Théologiques

Théologiques

\title{
Le dialogue islamo-chrétien au Québec : quelques signes d'espérance
}

\section{Samia Amor}

Volume 19, numéro 2, 2011

Le dialogue islamo-chrétien

URI : https://id.erudit.org/iderudit/1024731ar

DOI : https://doi.org/10.7202/1024731ar

Aller au sommaire du numéro

Éditeur(s)

Faculté de théologie et de sciences des religions, Université de Montréal

ISSN

1188-7109 (imprimé)

1492-1413 (numérique)

Découvrir la revue

Citer cet article

Amor, S. (2011). Le dialogue islamo-chrétien au Québec : quelques signes d'espérance. Théologiques, 19(2), 137-150. https://doi.org/10.7202/1024731ar
Résumé de l'article

Dans un contexte de mobilité humaine à l'échelle planétaire, l'implantation de l'islam au Québec est un fait de société qui entraîne une rupture avec la représentation classique des relations islamo-chrétiennes. On se demande comment envisager un renouvellement de la communication entre chrétiens et musulmans au Québec. Cette contribution s'attache à repérer les foyers de dialogue, ce qui rompt avec l'idée d'une absence de contact entre les deux communautés de croyants. Elle étudie la possibilité d'un Québec en tant qu'espace de dialogue, tout en s'interrogeant sur un cadre de référence pour un dialogue favorable à une cohésion sociale. 


\title{
Le dialogue islamo-chrétien au Québec: quelques signes d'espérance"
}

\author{
Samia AMOR** \\ Droit \\ Université de Montréal
}

Si le «à côté de» ne se transforme pas en "avec», il se transforme en "contre». (M. Buber, La vie en dialogue)

Le dialogue, en tant que produit d'interactions sociales, est une prescription religieuse pour les musulmans ${ }^{1}$. Une obligation et un devoir plutôt qu'une option. Le rapprochement entre croyants qu'il préconise hisse le dialogue au rang d'un absolu relationnel qui participe et façonne l'expérience humaine. L'échange intersubjectif fait partie de l'itinéraire spirituel qui mène à Dieu. Il constitue une occasion d'exercice à une pédagogie coranique tournée, justement, vers une éthique comportementale.

Certes, l'islam se traduit par un ensemble d'obligations individuelles et parfois collectives de culte ('ibadates) significatives d'une foi. Mais il repose essentiellement sur un mode de vie et un agir humain (mu'amalat) dans une temporalité conjoncturelle. D'emblée, une telle vision du dialogue contraste avec la conception assez généralisée, tant du côté musulman que du côté chrétien, d'un échange humain basé sur une «alternative» formulée en termes d'inclusion et d'exclusion.

\footnotetext{
* Je remercie les évaluateurs anonymes pour leur lecture attentive et les commentaires constructifs apportés à ce travail.

* Samia Amor est doctorante à la Faculté de droit de l'Université de Montréal, elle a récemment publié (2012) «L'islam et la commanderie du bien ou l'agir quotidien au Québec ", dans B. Demers, dir., Dialogue des cultures et traditions monothéistes, Montréal, Novalis, p. 175-182.

1. Coran XXIX, 46: «Avec les Juifs et les Chrétiens, ne discutez que de la meilleure manière ».
} 
À notre avis, bien au contraire, la perception islamique du dialogue situe celui-ci dans une égalité ontologique, appuyée par les récits heuristiques sur les prophètes et les peuples précédents. Elle l'envisage dans un mode de conciliation plutôt que de conflits. Et l'approche "herméneutique de soi » de Ricœur (1989, 14), dans lequel s'inscrit ce travail, en donne un écho. En effet, celle-ci ouvre sur une solution de rechange à «l'apologie du cogito cartésien ». Le "soi» établit une distance par rapport au «je», enfermé dans son égocentrisme, et libère la place à l'altérité. Il est surtout l'expression d'une autonomie animée par une éthique qui a pour souci d'intégrer autrui en tant que constitutif de sa propre identité. Et le dialogue se dégage d'une rencontre entre des personnes. En conséquence, apprendre à connaître l'autre, l'étranger à soi et grâce à qui on revient à soi, conduit progressivement à mettre de côté les clichés négatifs sur lui. Ce qui convie à abandonner la peur, la stigmatisation, voire le fantasme, à la faveur d'une reconnaissance et du respect de la dignité de l'autre.

Mais comment dialoguer «de la meilleure manière» avec les croyants des autres religions, voire avec tout autre humain simplement? Comment envisager un dialogue islamo-chrétien lorsque certains évènements aux niveaux national et international mettent l'éclairage sur une pratique de l'islam à l'effet refroidissant et dissuasif ? Le phénomène d'immigration musulmane au Québec prend de l'ampleur dans les années 1990. Une présence qui transforme la configuration architecturale (construction exponentielle de mosquées) et suscite une polémique dans l'espace public (voile et interdits alimentaires). La tendance « universelle » à la suspicion envers les musulmans soulève un rejet plutôt qu'un rapprochement. Comment, indépendamment de ces faits, se construit le dialogue islamo-chrétien au Québec?

Cet article s'attache à mettre en lien ces questions, à partir de trois angles: 1. l'émergence, depuis les années 1990, de foyers de dialogue islamo-chrétien au Québec; 2. l'actualité du contexte québécois sur celui-ci et 3. une perspective pour un rapprochement.

\section{1. État des lieux du dialogue islamo-chrétien au Québec}

Au préalable, le besoin de rapprochement entre les chrétiens et les musulmans est à relier à l'augmentation de la population musulmane au Canada, au cours des années 1980 (62 385 en 1980, 108000 en 2001 et 940000 
en $2010^{2}$ ). Auquel s'ajoutent les évènements tragiques du 11 septembre 2001, générateurs de sentiments d'inquiétude, de peur et de suspicion, exacerbés dans les pays d'Europe et d'Amérique du Nord, à l'égard des minorités musulmanes installées sur leur territoire. À tel enseigne que les mesures sécuritaires adoptées par ces pays ont créé l'archétype du musulman migrant ou de l'intérieur, ennemi opposé aux "valeurs judéo-chrétiennes». La combinaison de ces facteurs forme le terreau des enjeux politiques, électoraux et de débats publics. Les représentations de violence, de barbarie et de fanatisme sont mobilisées pour présenter l'islam comme «le» péril du XXI ${ }^{\mathrm{e}}$ siècle.

Effectivement, les évènements survenus au cours des dernières décennies, dans certains pays à majorité islamique (mouvements islamistes dans les pays du Maghreb et au Proche-Orient, attentats en France, en Espagne et à Londres, etc.), alimentent les sentiments de risque lié aux musulmans. Ils aident à la prolifération d'une littérature spécialisée qui renforce l'image négative des musulmans et de l'islam. Cela explique, sans la justifier, la frilosité à l'égard d'un quelconque dialogue avec les musulmans!

Toutefois, dans les années 1960, une «islamité québécoise », selon l'expression de Daher (2003, 29), s'est édifiée par vagues provenant de différentes régions du monde. Caractérisée par un pluralisme ethnique, linguistique et doctrinal (sunnite, shi'ite, kharédjite, confréries soufies), elle s'est mêlée à la société, sans controverses. D'ailleurs, au nom du droit de chacun à vivre sa religion, la loi 194, du 6 août 1965, reconnaît l'islam comme «religion d'une minorité ethnique». Désormais, l'enregistrement des mariages des musulmans ne relève plus des prérogatives des Églises.

En parallèle, l'un des effets heureux de cette poussée migratoire musulmane, qui se sédentarise en majorité une décennie plus tard, consiste en la mise en place d'un dialogue islamo-chrétien. Précurseur, ce dialogue émane d'initiatives institutionnelles chrétiennes. Le peu de résonnance rencontré du côté musulman s'explique-t-il par l'absence de magistère équivalent ? La carence dans l'exploration de cette question met en relief le fait, à un niveau informel, d'une mobilisation de croyants musulmans ordinaires concernés par un rapprochement de valeurs spirituelles communes de foi en Dieu et de piété3.

2. Ministère de l'immigration et des communautés culturelles du Québec $(2005,4)$ et Pew Forum on Religion and Public Life (2011).

3. La piété est entendue comme l'expression de l'engagement religieux, du croyant, au cours de ses interactions sociales. Elle reflète un comportement en conformité avec sa foi. 
L'expérience œcuménique des institutions chrétiennes les habilite, dès 1989, à créer un comité national de liaison pour un dialogue entre musulmans et chrétiens ${ }^{4}$. Il rassemble plusieurs organisations musulmanes et chrétiennes, telles que l'Église unie du Canada, la Conférence des évêques catholiques du Canada, le Conseil canadien des Églises, l'Église presbytérienne du Canada, le Conseil des imams, le Conseil canadien des femmes musulmanes, le Congrès islamique canadien, le Cercle islamique d'Amérique du Nord et le Conseil de la communauté musulmane. Dès 1996, une représentation du Québec rejoint ce comité national. Dans l'intervalle, en 1991, le Centre Afrika ${ }^{5}$ instaure une série de conférences et de colloques, en vue de promouvoir ce type de dialogue à travers l'ensemble du Québec. Ce même centre met en place, avec le concours de bénévoles, un projet «interreligion" qui se transforme, dès 2001, en trois groupes de dialogue: le Comité de dialogue entre chrétiens et musulmans du Québec, le groupe de rencontres spirituelles et le Cercle de dialogue.

De la pratique dialogale du Centre Afrika, naît l'instauration d'un espace de parole, appelé "Comité de dialogue entre chrétiens et musulmans du Québec ». Son organisation s'inspire de celle du Comité national de liaison du dialogue entre musulmans et chrétiens. Il fonctionne avec douze membres, recrutés par mode de nomination ou de cooptation, qui se réunissaient cinq à six fois par an. Son activité a pris fin en 2008. Son but était de «favoriser le rapprochement, la compréhension et la collaboration entre membres des communautés musulmanes et chrétiennes du Québec ». Pour atteindre son objectif, le Comité se voulait un réseau à trois voies: l'information sur le dialogue islamo-chrétien au Québec, le dialogue entre les membres et l'intervention sous forme de manifestations publiques, de signatures de pétition. Il se qualifiait $d$ ' "aviseur auprès d'instances institutionnelles qui le sollicitent ${ }^{6} »$.

Dans un registre similaire, le Cercle interreligieux, porté par quinze membres, se forme dès le 25 mars 2003. Il restera en vigueur jusqu'en 2008. Le groupe comprenait des responsables de centres, d'organismes ou d'ins-

4. Les opportunités de dialogue observées sur le terrain n'ont pas pour objet de dresser une nomenclature. Elles ne sont que des exemples parmi d'autres et ne prétendent à aucune exhaustivité.

5. Le Centre Afrika abrite dans ses locaux les rencontres de dialogue instaurées par le projet «interreligions », initié par Gilles Barrette.

6. Cet objectif est cité à l'article II c) des statuts du Comité musulmans-chrétiens du Québec, qui se retrouve sur leur site: <interreligion.mafr.net/comitéldialogue.html>, (dernière consultation le 24 mars 2013). 
tituts religieux. Son fonctionnement alternait entre rencontres mensuelles et conférences. Les réunions mensuelles, programmées de septembre à juin, avaient pour objectif d'instaurer une rencontre de croyants des deux traditions afin de parvenir à un rapprochement des cultures et à une réconciliation, au niveau international. Le dialogue portait sur des thèmes choisis avec textes à l'appui. Les conférences, les visites d'écoles ou de lieux de rencontre se faisaient à la demande.

Le troisième forum de dialogue piloté par le Centre Afrika, créé en février 2002, demeure encore fonctionnel. Il organise des rencontres spirituelles avec le concours de ses quinze membres. Les rencontres mensuelles, le premier dimanche de chaque mois, comprennent des échanges et des prières en commun. Il se fonde sur le dialogue de la foi.

L'engagement du Centre Afrika pour un rapprochement interreligieux a inspiré d'autres expériences de dialogue, parmi lesquelles le groupe Interfoi mis en place par le Carrefour Foi et Spiritualité, à Cartierville. Son mode de fonctionnement comprend des rencontres mensuelles, le troisième dimanche du mois. L'objectif vise à permettre aux membres de "partager leur expérience de Dieu à travers leurs traditions respectives». Dans la même foulée, et depuis 2010, l'unité pastorale constituée par les paroisses Saint-Arsène, Saint-Édouard, Saint-Étienne et la Mission Ste-Thérèse d'Avila abrite un groupe composé entre autres de musulmans et de chrétiens pour un repas et une prière en commun. En 2011, un groupe de féministes croyantes chrétiennes-musulmanes se réunit autour d'un objectif commun, l'égalité des femmes, au sein de leurs traditions religieuses. Le projet encore embryonnaire tient des rencontres trois fois dans l'année, autour des questions de justice sociale.

La disposition institutionnelle chrétienne au dialogue trouve écho auprès de l'imam Sayed Nabil Abbas, du Centre islamique libanais. Représentant du courant shi'ite musulman, il a encouragé les rencontres entre les deux communautés de croyants par l'organisation de conférences annuelles ${ }^{7}$. L'initiative est à souligner, même si la régularité fait défaut, par manque de relève. Encore une fois, l'absence de recherche dans ce domaine oblige à la prudence, quant à l'interprétation de cette lacune. Toutefois, la rencontre de jeunes croyants, en dehors du contexte scolaire ou universitaire, pourrait favoriser l'échange dans la diversité de la foi. Une autre

7. Colloque Femmes et religions abrahamiques au Québec, Montréal, 8-10 septembre 2011. 
initiative mérite d'être soulignée, celle de la Fondation Dialogue (Intercultural Dialogue Institute Montreal). Créée en 2003, par des Canadiens d'origine turque, elle promeut le dialogue et la tolérance entre les peuples et les religions. Dans cette perspective, elle multiplie les rencontres sous forme d'activités communes, comme les conférences suivies de repas et les voyages en Turquie. Dans une gamme similaire, le collectif «Présence musulmane à Montréal» a tenté de s’impliquer dans un dialogue citoyen ${ }^{8}$.

Même si, à notre connaissance, le nombre de lieux de dialogue au Québec semble, prima facie, infime, ce dialogue n'en demeure pas moins fécond. Il s'articule autour de valeurs communes, telles que la foi en Dieu, la moralité du comportement et la piété. Dès lors, comment le Québec pourrait-il abriter, à travers le dialogue islamo-chrétien, un espace commun pour Dieu, dans un environnement de plus en plus réfractaire à l'idée de Dieu et dans lequel la question religieuse reliée à l'islam sous-tend des enjeux politiques?

\section{Le Québec: espace de convivialité ou de conflit?}

Terre d'accueil et de pluralisme culturel, ethnique et religieux, le Québec doit faire face au défi d'harmoniser et d'équilibrer deux notions antithétiques: diversité et unité. D'une part, la diversité de la composante sociale, découverte comme une nouveauté, ces dernières années, malgré une réalité forgée depuis quatre siècles par une présence autochtone, anglaise, juive, chinoise, etc., paraît porteuse d'un risque d'éclatement de la société en de multiples microcosmes. Il en découle que le clivage social semble relever davantage de la différence religieuse que des inégalités socio-économiques. Un biais qui suscite scepticisme et même inquiétude. D'autre part, nous trouvons une uniformité revendiquée face à la visibilité religieuse, notamment celle de l'islam, perçue comme une menace pour la culture et pour la religion des uns et des autres. Comment concilier le droit à la différence et le droit à la préservation de ses repères culturels?

8. L'une des missions de ce collectif est de: «susciter et contribuer au dialogue, participer aux débats publics tout en prenant position sur les questions sociales et les problématiques touchant tant les Québécois de confession musulmane que la société québécoise dans son ensemble. [...] en privilégiant une approche qui encourage la prise d'initiative personnelle, la consultation mutuelle, l'esprit d'équipe, et les partenariats intra et inter communautaires. »Voir site:<www.presencemusulamne.org $>$ (dernière consultation le 24 mars 2013). 
Le Québec détient-il une capacité à favoriser un dialogue pacifique, entre les personnes et les groupes qui le composent? Peut-il pousser celuici à s'acheminer vers une position commune? Pouvons-nous penser ensemble la cohérence du changement? Pouvons-nous penser une action efficace pour une unité harmonieuse au fondement d'un avenir commun, déterminé par une solidarité humaine, une justice sociale et une responsabilité? Dans une société de pluralisme religieux, comme le Québec, l'entente sociale a pour exigence la séparation des institutions politiques et religieuses, une séparation caractérisée par une neutralité dans le fonctionnement. Cette neutralité se traduit par l'absence de toute préséance de valeurs, de rites et de croyances d'une tendance religieuse particulière.

Or, consécutivement à la "crise des accommodements ", survenue en 2007, des discours se développent en s'articulant autour de confusions, ignorées ou entretenues, sur la notion de laïcité. Au préalable, la controverse sur les accommodements estompe le fait que ces derniers sont un moyen d'application des principes juridiques contenus dans les chartes québécoise et canadienne. En ce sens, il n’y a rien de déraisonnable, si ce n'est le malaise identitaire sous-jacent. Or, ce malaise se manifeste par une dichotomie entre le «nous » québécois catholique et le "eux», ces immigrants musulmans, quelle que soit leur appartenance (sunnites ou chi'ites). Contrairement à ce qui est présumé, ces derniers demeurent sensibles aux aspirations d'indépendance et de souveraineté inclusive des Québécois. Mais comment peuvent-ils y contribuer par leur propre expérience de reconquête d'un pays précédemment perdu, lorsqu'ils sont marginalisés par le discours d'opposition?

Bien plus, en mobilisant la laïcité pour affirmer une identité québécoise, une certaine rhétorique en cours ${ }^{9}$, favorable à une implication de l'État, contribue à brouiller la compréhension de cette notion. Avec l'amalgame des deux notions, laïcité et identité, l'altérité de l'islam est surtout perçue comme une menace à conjurer préventivement plutôt que comme une

9. En fait, la crise des accommodements raisonnables a mis en évidence un rapport entre la laïcité et l'identité québécoise, selon le rapport de la commission Bouchard-Taylor. Cet aspect génère un débat sur le type de laïcité appropriée au Québec et donne lieu à une controverse entre les partisans d'une laïcité ouverte à la présence de la religion dans l'espace public (exprimée dans le Manifeste pour un Québec pluraliste) et les défenseurs d'une laïcité " tout court» (soutenue dans la Déclaration des intellectuels pour la laïcité). En conséquence, et à défaut de la promulgation d'une charte sur la laïcité, le gouvernement québécois a inclus une déclaration sur le respect des valeurs québécoises, dans le certificat de sélection des immigrants. Voir l'analyse de Labelle (2012) pour saisir les enjeux en présence. 
relation de rencontres. Conséquemment, le débat s'éloigne du sens initial de la laïcité. Or, conçue comme un programme politique, celle-ci cherchait à mettre fin à l'intolérance religieuse à l'égard du judaïsme et du protestantisme et au conflit qui opposait l'État à l'Église vis-à-vis du statut du catholicisme comme religion structurante. Dans ce cadre, l'individu occupe le centre et organise sa propre réalité. Il dispose de garanties de l'État en matière de liberté de conscience et de liberté de religion. À travers la laïcité, l'État confère aux citoyens un cadre commun de pacification qui ne comporte ni l'exclusion de la religion de l'espace public ni celle de ses expressions symboliques. Cependant, la controverse contemporaine au Québec, autour de la laïcité, postule l'existence d'un communautarisme plutôt que d'un individualisme qui motiverait les pratiques religieuses, particulièrement celles de la tenue vestimentaire des musulmanes ou le sacrifice du mouton. Selon certains discours, la manière la plus efficace de le contrarier et de rassurer la société passerait par une législation préventive et sécuritaire.

Dès lors, la référence à l'islam dans les propos tenus pose l'exigence d'une intervention de l'État dans l'espace public. La laïcité aurait pour corolaire une posture de neutralité de l'État, autrement dit, la mise en retrait de l'État de l'espace public, sa gouvernance politique, sans privilèges à l'égard d'une religion quelconque, et une action dans l'intérêt public plutôt que pour des intérêts privés. Ce serait seulement de cette façon que la séparation entre le religieux et le politique prendrait sens. Toutefois, dans ces discours, la place de l'islam dans l'espace public est devenue objet de controverse, car elle pose la laïcité comme valeur inaltérable, au même titre que l'égalité homme-femme.

En comparaison avec la politique française à l'égard de l'islam et des musulmans, la tendance du discours québécois pour une laïcité d'intervention semble vouloir instaurer une mise sous tutelle de la religion et une définition de l'acceptable musulman dans l'espace public. Conséquemment, la liberté de religion, à l'origine du principe de laïcité, se ratatine au profit de l'égalité. Une suprématie clamée qui va justement à l'encontre du principe de non-hiérarchisation des droits fondamentaux, considérée par Gaudreault-Desbiens comme une "vérité apodictique (c.-à-d. "qui a une évidence de droit") » $(2012,7)$. D'ailleurs, l'auteur rappelle que le principe trouve une confirmation devant la Cour suprême canadienne, avec l'arrêt Dagenais qui précise qu'en cas de conflits entre droits fondamentaux, la règle est une recherche d'équilibre entre eux. Au Québec, le Tribunal des droits de la personne adopte postérieurement la même position. 
En bref, dans un tel contexte de piétinement du discours sur la laïcité, le projet souverainiste connaît un ajournement. Pourtant, ce projet est avant tout émancipateur de toute domination, pluriel et inclusif; en lui, chaque Québécois musulman se sent non seulement concerné, mais impliqué afin de se sentir chez lui, au Québec. En attendant l'adoption d'une définition de la nation québécoise inclusive de toutes ses composantes sociales: autochtone, d'immigration francophone et anglophone, ancienne et récente, quelle place accorder au dialogue islamo-chrétien?

La controverse autour de certaines questions, comme le voile, l'égalité homme-femme ou encore les salles de prière, risque de créer des impasses au dialogue entre majorité et minorité, impasses qui se reflètent dans la relation entre chrétiens et musulmans. Peut-on transcender ces signes de découragement et envisager avec espérance une prise de conscience sur ce qui est essentiel et concevoir une nouvelle forme de dialogue?

\section{Contribution à un dialogue de rapprochement}

Comment parvenir à un dialogue islamo-chrétien sincère, lorsque la relation entre musulmans et chrétiens porte déjà les stigmates de dissensions et d'inimitiés? Pourtant, tant le christianisme que l'islam disposent d'un vivier d'éthique, c'est-à-dire d'un ensemble de normes relatives aux devoirs et aux responsabilités du croyant envers autrui, croyant ou non. D'emblée, celles-ci invitent à l'édification de l'unité, quelle que soit la diversité de la société. Ces exigences, souvent négligées, voire trahies, dessinent la perspective d'un vivre-ensemble qui n'est ni vivre à côté, à l'écart, ni vivre de son côté, dans l'indifférence de l'autre, mais vivre côte à côte, ensemble. Dès lors, il devient impératif, dans le Québec contemporain, de puiser dans ce vivier à identifier pour chaque groupe.

La connaissance est une condition préalable à la démarche d'aller vers l'autre. Du côté musulman, il faut en apprendre plus sur le credo chrétien et la place de Jésus, et, surtout, se défaire de cette certitude d'impiété ancrée dans l'imaginaire de certains. D'ailleurs, être en désaccord avec la vision trinitaire chrétienne n'exclut pas le respect de celui qui l'exprime, de sa manière de vivre et d'agir dans sa foi. Cette fides ou confiance, au-delà de la divergence dans la compréhension de la conception de l'unicité divine, incite au rapprochement pour en saisir les nuances. Chacun des groupes a des choses à dire et à entendre de l'autre. Il n'est pas nécessaire d'être d'accord sur les questions théologiques ou encore de poser l'obligation d'y adhérer pour nouer une relation tissée dans la trame de l'amitié. Cette 
dernière, un don de Dieu à partager, facilite la discussion des différences avec sincérité et confraternité.

Du côté chrétien, la connaissance de l'islam et des musulmans dispose au dépassement de la représentation fantasmée, guerrière et suspicieuse, véhiculée depuis le Moyen-Âge. Elle favorise la conversion du regard sur une réalité vécue par des personnes de proximité plutôt que sur des situations rapportées dans les médias, relais d'évènements lointains. C'est au risque des rencontres que se révèle l'humanité de l'autre.

Dans ces croisements de regards sans croisades, le décloisonnement religieux favorise un authentique dialogue dénué de marqueurs religieux, identitaires, de surdité, et doté d'une autre vision. Dans le face-à-face d'humains, la communication, c'est-à-dire « une relation dramatique dans laquelle tour à tour je m'affirme dans mon origine et je me livre à l'imagination d'autrui selon son autre civilisation » (Ricœur 1961, 453), devient garante de la cohabitation. Bien plus, la communication encourage la conception de projets communs pour une expérience commune transformatrice. Elle invite à la rencontre, celle qui encourage à aller vers l'autre et à le laisser venir vers soi. Sans occulter les difficultés de compréhension ni s'y attarder, au risque de paralyser l'interaction, les perspectives d'un véritable dialogue exigent un préalable: la réflexion sur une éthique de ce dialogue. En effet, le dialogue ne se vit pas seul, mais à deux ou plus. Même s'il dépend de l'histoire et de la culture de chacun, il est fondé sur une égalité des partenaires. La connaissance s'édifie autour de quatre références, à mon avis, cruciales pour la réussite d'un dialogue: l'attitude, la vigilance, la reconnaissance de l'autre en tant qu'humain et en tant que produit d'identités multiples.

Premièrement, le dialogue est un état d'esprit, une attitude. Il confère à celui qui le pratique l'aptitude à écouter, à chercher à comprendre, à connaître et à se défaire progressivement des préjugés. L'intention se dépouille de toute velléité de conversion pour apprendre de l'autre uniquement.

Deuxièmement, les partenaires du dialogue sont invités à être attentifs à la violence insaisissable, insidieuse et aux allures agréables. Celle qui prend la forme, soit d'une pédagogie sur la religion de l'autre, soit de l'usage d'un ton paternaliste ou encore celle qui encourage l'apologie de sa propre religion. Elle met une religion - en l'occurrence, l'islam - sous les projecteurs, avec des arguments à charge, dès les premiers échanges. Le dialogue vire aisément à la confrontation et laisse croire que tout est mobilisé pour le neutraliser justement. 
Troisièmement, la reconnaissance de l'autre revêt la forme du respect dans la manière de lui parler, de l'écouter et de le dénommer. Même si chaque religion a prétendu, dans le passé et encore aujourd'hui, détenir le monopole de la vérité et du salut, il faut, avec intelligence, éviter le piège de la dénégation qui risque de poser sur l'islam un regard chargé de supériorité. Ce qui conduit au respect d'une réalité indéniable, le pluralisme religieux, et qui plus est, dans la résonnance de sa dimension égalitaire. Cette reconnaissance ouvre sur une approche de cordiale altérité qui rejette toute déconsidération de l'autre, en raison justement de sa religion. Elle appelle à un dépassement, celui de l'essentialisme, pour toucher à l'essence de l'autre. Cette interaction avec l'extérieur à soi constitue la condition sine qua non de la constitution de soi. Et cette recherche de la connaissance de l'autre peut entraîner une véritable amitié, celle qui ne cherche pas à convertir, mais qui accepte l'autre, tout en lui attribuant une opinion sincère. Une amitié qui cherche plutôt à briser les murs dressés de la suspicion et à ouvrir le cœur à la spontanéité et à l'amour.

Quatrièmement, le regard positif posé sur le partenaire du dialogue stimule la prise de conscience des multiples facettes de l'identité d'un individu. Ce qui l'extrait de l'enfermement dans une seule de ses composantes, notamment la dimension religieuse. Elle ravive la mémoire que l'identité n'est ni figée ni définitive. C’est un processus qui se déploie avec le temps et au gré des expériences humaines.

Ces conditions, puisées dans les expériences personnelles de dialogue, favorisent une communication, au sens donné par Ricœur, une communication construite à l'abri de l'apologétique et du polémique afin de parvenir à un dialogue sincère. Cette communication pose comme exigence l'écoute de l'autre afin de sortir des préjugés le concernant et des quiproquos qui séparent. Elle interroge sur les points de convergence, mais également sur les questions qui dérangent, afin d'éviter de rester dans un échange aseptisé et courtois. Autrement dit, elle nécessite la mise en place d'une éducation aux relations sociales, orientée vers un vivre-ensemble de transformation mutuelle. Une telle approche met aussi en place une éthique, c'est-à-dire la «recherche de la vie bonne, avec et pour autrui dans des institutions justes » (Ricœur 1989, 202). Elle invite, par le rapprochement, au témoignage réciproque de la présence de Dieu. Dès lors, le respect, la reconnaissance de la différence, la racine spirituelle commune sont, avec la diversité ethnique, linguistique et religieuse, un don divin qui nécessite le dialogue. 
En conclusion, l'approche du dialogue par les minorités de croyants, qu'ils soient chrétiens ou musulmans (mais également juifs, pour ne parler que des adeptes du monothéisme) ouvre un espace d'échange d'expériences individuelles qui se rejoignent et un espace commun pour Dieu. Il permet la prise de conscience que les traditions religieuses sont enracinées les unes dans les autres, même si elles empruntent différentes voies. L'arbre généalogique commun, au-delà du partage scripturaire, indique que l'humain ne peut vivre seul et qu'il a besoin de l'autre pour cheminer dans la voie de Dieu. Comme le soulignent de Pury et Macchi $(2004,10)$ :

Chacune des trois [religions] a tendance à se considérer comme la quintessence des deux autres: le judaïsme parce qu'il se sait la racine, l'islam parce qu'il se voit comme l'aboutissement et le christianisme parce qu'il en exprimerait le secret. Cette rivalité fait figure d'aveu: en dépit des apparences, aucune des trois religions ne peut se passer des deux autres. Chacune a besoin des autres pour rendre compte de son identité propre.

Une telle compréhension de l'imbrication des religions conduit à considérer la religion du partenaire du dialogue comme une partie intégrante de sa croyance.

\section{Références}

Arrêt Dagenais contre Société Radio-Canada [1994], 3 R.C.S., 835, par. 72. Buber, M. (1959), La vie en dialogue, trad. par J. Loewenson-Lavi, Paris, Aubier.

Centre Afrika, <http://www.interreligion.mafr.net> (consulté le 24 mars 2013).

C.D.P. contre Centre d'accueil Villa Plaisana, T.D.P.Q. Gaspé, 1995, CanLII 2814 (QC T.D.P.), p. 21.

Daher, A. (2003), «Les musulmans au Québec », Relations, juin, p. 29-32.

Fondation Dialogue (Intercultural Dialogue Institute Montreal), <http://montreal.interculturaldialog.com> (consulté le 15 novembre 2012).

Gaudreault-Desbiens, J.-F. (2012), «Les hiérarchies passagères, ou de la contingence dans l'équilibrage entre droits fondamentaux", Revue québécoise de droit constitutionnel, p. 7-41.

LABelle, M. (2012), «L'enjeu de la laïcité à la lumière du débat sur l'identité et le pluralisme culturel », Vivre Ensemble, 19/65, p. 1-6. 
Grand dictionnaire Larousse encyclopédique (1982), Paris, Larousse.

Le Carrefour Foi Et Spiritualité (Cartierville), <http://foi-spiritualite. ca/?s=interfoi> (consulté le 12 août 2012).

Ministère De L'ImMigration et Des COMmunautés Culturelles du QuÉBEC (2005), Portrait statistique de la population d'origine ethnique arabe, recensée au Québec en 2001, Québec.

Petitclerc, J.-M. (2004), Et si on parlait... du suicide des jeunes, Paris, Presses de la Renaissance.

Pew Forum on Religion and Public Life (2011), "The Future of the Global Muslim Population: Projections for 2010 to 2030 », 27 janvier, <http://pewforum.org/The-Future-of-the-Global-Muslim-Population. aspx> (consulté le 24 mars 2013).

Pury, A. de et Macchi, J.-D. (2004), dir., Juifs, chrétiens et musulmans. Que pensent les uns des autres, Genève, Labor et Fides.

PrÉSENCE Musulmane, <www.presencemusulamne.org> (consulté le 24 mars 2013).

Ricceur, P. (1961), "Civilisation universelle et cultures nationales ", Esprit, 29/10, p. 439-453.

(1989), Soi-même comme un autre, Paris, Seuil.

Statistique Canada (2003), Recensement de 2001 : série "analyses", les religions au Canada, catalogue n96F0030XIF2001015, 13 mai.

\section{Résumé}

Dans un contexte de mobilité humaine à l'échelle planétaire, l'implantation de l'islam au Québec est un fait de société qui entraîne une rupture avec la représentation classique des relations islamo-chrétiennes. On se demande comment envisager un renouvellement de la communication entre chrétiens et musulmans au Québec. Cette contribution s'attache à repérer les foyers de dialogue, ce qui rompt avec l'idée d'une absence de contact entre les deux communautés de croyants. Elle étudie la possibilité d'un Québec en tant qu'espace de dialogue, tout en s'interrogeant sur un cadre de référence pour un dialogue favorable à une cohésion sociale. 


\section{Abstract}

In the context of human's mobility in the world, Islam's settlement in Quebec becomes a social fact, which breaks from traditional representations of Christians and Muslims relationships. Hence, we ought to wonder how is it possible to renew the communication between the two communities? This paper aims at identifying kinds of dialogue that break away from the belief that both communities are normally disconnected. We envision Quebec as a forum for dialogue meanwhile questioning a possible exchange framework that would facilitate a greater social cohesion. 